\title{
Multiplex ligation-dependent probe amplification: a novel approach for genetic diagnosis of Porphyria
}

\author{
Elena Di Pierro, Valentina Brancaleoni, Valeria Besana and Maria Domenica Cappellini \\ Porphyrias are disorders caused by the genetic defects of enzymes of the heme pathway and are characterized by such a wide \\ genetic heterogeneity that even the molecular analysis is not always decisive for a correct diagnosis. In the past few years, \\ deletion with a size range of few kilobase pairs have been reported. These peculiar mutations, missed by both sequencing and \\ cytogenetic techniques, have been identified by time consuming and technically demanding methods. To provide a rapid and \\ sensitive method for the detection of deletions responsible for porphyria, we successfully designed and tested seven chemically \\ synthesized probe sets specific for each heme gene and their flanking regions, to be used in multiplex ligation-dependent probe \\ amplification technique.
}

Journal of Human Genetics (2009) 54, 479-487; doi:10.1038/jhg.2009.67; published online 24 July 2009

Keywords: deletion; MLPA; porphyria

\section{INTRODUCTION}

Porphyrias (mim 125270, 176000, 263700, 176100, 121300, 176200 and 177000) are rare diseases caused by genetic defects of the enzymes involved in the heme pathway (EC: 4. 2.1.24, 2.5.1.61, 4.2.1.75, 4.1.1.37, 1.3.3.3, 1.3.3.4, and 4.99.1.1, respectively). Porphyrias are classified as 'acute' and 'chronic' based on their clinical manifestations, or as 'hepatic' and 'erythropoietic' according to the two main sites in which the excess metabolites are produced. ${ }^{1}$ Acute porphyrias are characterized by mild to severe acute attack with neuromuscular dysfunctions affecting the autonomic, peripheral or central nervous system, that can lead to death if not detected. In approximately $10 \%$ of cases, acute liver failure has been reported. ${ }^{2,3}$ Chronic Porphyrias are characterized by mild to severe cutaneous photosensitivity and chronic involvement of the liver with increased risk of hepatocarcinoma. ${ }^{4}$ Nowadays, the diagnosis of Porphyria is based on clinical symptoms supported by specific pattern of porphyrins in blood, urine and stools; however, because of physiological fluctuations, the measurements of porphyrinic metabolites are not always reliable. ${ }^{5}$ The erythrocyte enzymes' activity confirms the diagnosis. However, the normal and pathological values might overlap, becoming misleading. ${ }^{6}$ Genetic analysis would be the only reliable tool to establish a correct diagnosis of Porphyria even though the high genetic heterogeneity makes this approach difficult. Although restricted geographic areas seem to have a high prevalence of specific molecular abnormality, ${ }^{7}$ most of the reported mutations have been detected in a single case or a family. ${ }^{8-10}$ Moreover, approximately $10 \%$ of porphyria cases remain devoid of molecular diagnosis. The reported molecular abnormalities of the different forms of Porphyrias are so far limited to point mutations or small deletions/insertions in the coding regions and in the splicing junctions of the seven genes involved in the heme pathway: ALAD (GeneID: 210); HMBS (GeneID: 3145); UROS (GeneID: 7390); UROD (GeneID: 7389); CPOX (GeneID: 1371); PPOX (GeneID: 5498); and FECH (GeneID: 2235), which are readily detected by PCR and sequencing techniques (http://www.hgmd.cf. ac.uk/ac/index.php). Recently, single gene or long portion gene deletion have also been reported. ${ }^{11-13}$ These peculiar mutations of the size of a few kilobase pairs are missed by both PCR and by cytogenetic techniques, including fluorescent in situ hybridization. Long PCR, absence of Mendelian segregation of polymorphisms and gene dosage, commonly used to identify large deletion, are time consuming and technically demanding. Moreover, the feasibility of these techniques depends on the availability of DNA from biological parents, good quality DNA, and polymorphic sites with high frequency and efficiency of multiplex PCR. ${ }^{14,15}$ For all these reasons, it is possible that long gene deletions have gone undetected in patients with clinical symptoms and biochemical signs of porphyria. Recently, a rapid and relatively easy technique for DNA quantitative analysis, named multiplex ligation-dependent probe amplification (MLPA), has been described. ${ }^{16}$ This method, based on the hybridization and ligation of two adjacently annealing oligonucleotides, is suitable for detecting single gene or long sequence deletion. Each oligonucleotide pair (probe) is designed to have common ends, which means that all probes can be simultaneously amplified with one universal primer pair. By using a single fluorescently labelled universal primer, the resulting products can be easily separated based on size and then quantified. To improve the molecular diagnosis of porphyria and to 
simplify the detection of deletions in the heme genes, we designed and tested seven chemically synthesized probe sets specific for each heme gene and their flanking regions covering genomic regions from 39 to $152 \mathrm{~kb}$. In this paper, we report the validation of the MLPA using our set of probes and we confirm that MLPA is a very useful and reproducible method for improving molecular diagnosis of Porphyria.

\section{MATERIALS AND METHODS}

\section{Probe design}

A total of 144 probes were designed to detect the rearrangements on the heme genes: 16 for ALAD; 23 for HMBS; 20 for UROS; 18 for UROD; 24 for PPOX;
16 for CPOX; 24 for FECH; and 3 for internal control genes (ILKAP, DACH and DEFB129). The sequences of the probes are shown in Table 1. The probes were split into 7 sets, each containing the specific probes for a single heme gene and 3 control probes for a maximum of 27 probes. Each probe sets covered the following genomic regions, respectively: gene ALAD: NC_000009:115174000115213000 (reverse complement); gene HMBS: NC_000011:118442000118494000; gene UROS: NC_000010:127443000-127533000 (reverse complement); gene UROD: NC_000001:45225000-45320000; gene CPOX: NC_000003: 99733000-99863000 (reverse complement); gene PPOX:NC_000001: 159334500-159451000; gene FECH: NC_000018:53290000-53442000 (reverse complement). These regions contained at least the target gene with upstream and downstream genes. In the presence of small genes, close to one another,

Table 1 Names and sequences of probes

\begin{tabular}{|c|c|c|c|c|}
\hline & Name & Upstream hybridizing sequence & Downstream hybridizing sequence & s.d. \\
\hline \multicolumn{5}{|c|}{ ALAD } \\
\hline 1 & POLE3_4 & CTCAGTGCTAACAACTTTGCAATGAAAGGAAAGCGGAAGACG & CTGAATGCCAGTGATGTGCTCTCAGCCATGGAAG & $0.08(0.90-1.14)$ \\
\hline 3 & ALAD_2 & GGAGTTAACACACCAGGGTTCTAGT & CCTTTTGCTCGCTGTGTGGCT & $0.05(0.94-1.08)$ \\
\hline 4 & ALAD_3 & TCСААССТСАТСТАССССАТСТTTG & TCACGTGAGTCTCCAAGAATGGG & $0.06(0.88-1.03)$ \\
\hline 5 & ALAD_4 & СTTCCCACAGCTACCAATCCATATCCCACCCCCGCTCTTGCAG & GGATGTTCCTGATGACATACAGCCTATCACCAGCC & $0.05(0.90-1.03)$ \\
\hline 8 & ALAD_7A & GCTCCCCAAAACCCAGTCATCTG & TCCTGAAGGGCTCCTGAGTGAAAACGG & $0.06(0.86-1.02)$ \\
\hline 9 & ALAD_7B & CAAGGCAGGTGAGTGAACCACCAGCAGGGATGGGCACC & TCTGGGTCAGGAGGTGGCAGAGTG & $0.11(0.92-1.21)$ \\
\hline 10 & ALAD_8 & GATGTCAGGTGGTAGCCCCGTCGGACATGATGGATGGACG & CGTGGAAGCCATCAAAGAGGCCCTGATG & $0.07(0.88-1.10)$ \\
\hline 11 & ALAD_9 & CATAGGTATCGGTGATGAGCTACAGTGCCAAATTTG & СTTCCTGTTTCTATGGCCCTTTCCGGTGAG & $0.05(0.90-1.06)$ \\
\hline 12 & ALAD_10 & CACCCTGATGACTCTGCTTTGCAGG & GATGCAGCTAAGTCAAGCCCAGCTTTTGG & $0.09(0.86-1.14)$ \\
\hline 13 & ALAD_11 & GACATGCTCATGGTGAAGCCGGGAATGC & CCTACCTGGACATCGTGCGGGAGGTAAAGG & $0.01(0.97-1.01)$ \\
\hline
\end{tabular}

\section{HMBS}

1 VPS11_1

2 VPS11_6

3

VPS11_16

HMBS_1

HMBS_2

HMBS_3

HMBS_4

HMBS_5

HMBS_6

10 HMBS_7

11 HMBS_8

12 HMBS_9

13 HMBS_10

14 HMBS_11

15 HMBS_12

16 HMBS_13

17 HMBS_14

18 HMBS_15

19 DPAGT1_9

20 DPAGT1 4

21 DPAGT1_1

22 TMEM_1

23 TMEM_14

\section{GCCACACCTGCTTCTGGATCCGCTGCTTCCAAGTTCCTTTGC} CTGTGCAACAAGTTCATAGCCTATAGCACCGTCTTTGAGG

\section{CTGGCTGACATGCCCAGGGCTCCACTCTCATCT}

CACCCACACACAGCCTACTTTCCAAG

CTGAGGAGGGCAGAAGGTACTGAGGAAGGTTAAAGG

CAGGAAGAAAACAGCCCAAAGATGAG

CAGCTTGCTCGCATACAGACGGACAGTGTGGTGGCAACA

CTCTCTCCTCAGTTGCTATGTCCACCACAGG

CTGTCCGGCAGATTGGAGAGAAAAGCCTGTTTAC

CCATCTCTATAGAGTGGACCTGGTTGTTCACTCCTTGAAGG

GGGAAAACCCTCATGATGCTGTTGTCTTTCACCCAAAA

GTAACTTCTCTCTGGGCAGTGTGGTGG

CCGACACTGTGGTCCTTAGCAAC

CAGATCCTGCACCCTGAGGAATG

CATCTTGGATCTGGTGGGTGTGCTGCACGATCCCGAGA

GGCTGCAGTGTGCCAGTAGCCGTGCATA

CTGGAGGAGTCTGGAGTCTAGACGGCTCAGATAGCATAC

CATGAAGATGGCCCTGAGGATGACCCACAGTTGGTAGG

CAATGATCAAGGGACTCAGACATCATAGAA

CTCTACCAGGTTGAAGACAATGATGGAAGCAGA

AATGACTAGTGACTGGCCA

CCTCTCTAAGGCAACCTATGTTCTGCCCCGCTGCACCCGCCTA

CTCTTCGCCTTCAAGTCTTTCCGGGAGAACTGGCAGCG

GGCTTGGGTGCGAGC

CTTTTCTATTTATATGTGTGGCTTAGGACCCTCCGTGAA

CAGATGATAG
CTCCCTCCTGGCATCACTGTCTGCGACTCAGG

ATGTAGTGGATGTGCTTGCTGAGTGGGGCTCCCT

GTACGTGCTGACGC

AATGTCACAGCCCTCAGAACTAAAGCG

CGGAGCCATGTCTGGTAACGGCAATG

GACCAGCCTTGGAGTATTTCCCCACTCTGAGACTCAGCTGGC

AGTGATTCGCGTGGGTACCCGCAAGAGC

TTGAAAGCCTCGTACCCTGGCCTGC

GGACAAGATTCTTGATACTGCACTCTCTAAG

CAAGGAGCTTGAACATGCCCTGGAGAAGAATG

ACCTGCCCACTGTGCTTCCTCCTGGCTTCAC

TTTGTTGGGAAGACCCTAGAAACCCTGCCAGAGAAGAG

GAACCAGCTCCCTGCGAAGAG

TCTCCACAGCGGGGAAACCTCAACACC

CATGTATGCTGTGGGCCAGGTAC

CTCTGCTTCGCTGCATCGCTGAAAGGGCCTTC

CAGCTATGAAGGATGGGCAAGTAAGTGG

AAGAGACCATGCAGGCTACCATCCATGTC

CATCACTGCTCGTAACATTCCACGAGGGCCCCAGTTGGCTGC

GAGTCGAACGAGCTGATATCGAATGGAG

GCCTCTAGGCCGTTAATTCCTGCTAGGATATTGATGGC

TCTACTGTTTCCGCCCGGATCTTGTTCGCTGAACAACCATTAC GCTGAACGAGCAGGCCTGCAGAAACGGGGTGAGTTGGAC

AGGGCATCTCTCCCAGGTGACCCTTCTTTTCTGTC
$(0.94-1.07)$

$0.03(0.98-1.05)$

$0.02(0.99-1.05)$

$0.03(0.97-1.04)$

$0.04(0.96-1.06)$ $0.03(0.98-1.06)$

$0.04(0.96-1.07)$ $0.04(0.93-1.05)$ $0.04(0.96-1.06)$ $0.02(0.98-1.05)$ $0.04(0.99-1.10)$ $0.03(0.98-1.07)$ $0.05(0.93-1.07)$ $0.04(0.94-1.07)$ $0.03(0.98-1.05)$ $0.04(0.91-1.04)$ $0.03(0.99-1.09)$ $0.01(0.98-1.02)$ $0.03(0.95-1.06)$ $0.06(0.95-1.12)$ $0.03(0.97-1.06)$ $0.07(0.92-1.13)$

$0.03(0.98-1.08)$ 
Table 1 Continued

\begin{tabular}{lll}
\hline \multicolumn{2}{l}{ Name } & Upstream hybridizing sequence \\
\hline \multicolumn{1}{l}{ UROS } & \\
1 & BCC1P_8 & CTTATTATGACAGAGCAATTCCAACAGTCATGAAGGTTCC \\
2 & DHX32_8 & CAAATTGCTTTTCACATGTGCCACATGGAGCTGAAGAG \\
3 & BCC1P_6 & CTTCCACAAATGTCTTACTAATCAGAAGGTAAAAGTAG \\
4 & BCC1P_3 & CACACTCCCAATATGGTTCTGTTGAATTAAGAGATCTG \\
5 & UROS_Prom & CTTACAGTCACGTCCAGCTTGC \\
6 & UROS_IVS1 & CAGTAAAAGGGTGACTGTTGTTATTGCTGA \\
7 & UROS_2 & CTGCCAGGCAATAATGAAGGTTCTTTTACTGAAG \\
8 & UROS_3 & CCAATCCAGAACTATATTCTTTCATTTCAGGAATTAGGAT \\
& & TATATGGACTT \\
9 & UROS_4 & CTTTCTCATCCTGAAGATTACGGGGGACTCA \\
& & TTTTTACCAGCCCCAG \\
10 & UROS_5 & CAGTCTGGGAAAGGTCTCTGAAAGAAAAATGGATGC \\
& & \\
11 & UROS_6 & CCAGTGAGTAAAATTGGCCTGGATAC \\
12 & UROS_IVS6 & GGATTAGGACCCAGTTCTCTTGTTC \\
13 & UROS_7 & CTCAGCACTGCCTCTTCTATTTCCCTGTG \\
14 & UROS_8 & GTAAAGTCTTTCCTGCTTTTGATTGTGCCCAG \\
15 & UROS_9 & CTCTGGCCTCACATACAGTCTCAAGCACATTCAG \\
16 & UROS_IVS9 & CATCATGTGAGCATTCATTGACACAGG \\
17 & UROS_10 & CTCTGTGGAGCCAGCTTAAACC \\
18 & MMP21_IVS1 & GGGACAGCCCTGCAAGGTTCCCCAACACCGCAATAG \\
& & TAAGGATC \\
19 & MMP21_5 & CAGCTGGTACTGGCTTTATGAAAATCGAAACAATAGGACAC \\
20 & MMP21_7 & GAAAACCGGATTTTCAGTAGCTGAAGAAAATATGGCACTG \\
& & TAAGTTAAAAC
\end{tabular}

UROD

1 HECTD3_21 GAGGGAGGAACGGTAAACTGGGGCAGGGGTG TGCATAGCTCTAG

2 HECTD3_11 CGAGTAAGTCCCTCACTCTCATGCCATACCTGTAGTC

3 HECTD3_5 CTGTCCTGCCCAGAGCCTACTTACTTGACAATGGTGC

4 UROD_1 CAGATTCAGGTTAAATTGTGGATTGAGCTCGCAG TTACAGACAG

UROD_2 GAAACAGACTACACTCCCGTTTGGTGCATGCGCCAG

UROD_3 CAGGACTTTTTCAGCACGTGTCGCTCTC

UROD_4 CTGCCACCTAGCAACCTGTCTCCTG

UROD_5 GGATCCAGAAGTGGTAGCCTCTGAG

UROD_6 CCATCTTTCTATCCTTCTCTAGTGGACCCT

10 UROD_7 GCATTGCAGCTGTTTGAGTCCCATGCAGG

1 UROD_8

UROD_9

UROD_10

CGGTAAGCCATGGAAGGGTGAGG

GCTGGCTTTGCTTCCAGGGAGTGTGTG

GGAGGAGATCGGGCAGTTGGTGAAG

ZSWIM5_14 GAATTGTTCGGAGTCTCAAAGTGTCTCCTATAG

ZSWIM5_13 GTTGGGCAGGCAGACGGGTGATGCTGGAGCTTAAG

16 ZSWIM5_IVS8 GCAAATTCTTATTTGAGGTTGCTGTATCACAACTG AGTATACATG

17 ZSWIM5_7

GCACACCCATAAAGCCCCTGGAAAATAAAGCAAC CCATATTC

18 ZSWIM5_IVS5 GTCTGGTCAGCCTCTCCCACTAGATTTCAAGTAT TCAGTTCAG
Downstream hybridizing sequence

s.d.
CTGGAAATCACTTAGAGTTGATCTTGATTGAATTAGTC GCTGCCTTGACTTGTTGGAAGACATTTTTACATCCC CACTTCCCACATGGCTTATTGGTTCTGTGTGC

TTAGTTCTGCAGTGTTCACAGGAGCC

GGTCCCATCTTGCTTTTTTCTTCC

TACCCAGAACACCATGACATAGAAGG

GATGCGAAGGAAGATGACTGTGGCCAGGATCC

GAAGCCACTTTGATCCCTGTTTTATCGTTTGAGTTTTTGTC

AGCAGTGGAAGCAGCAGAGTTATGTTTGGAGCAAAAC

CAAGTCAGTGTATGTGGTTGGAAATGCTACTGCTTCTCT AGGTAAGGAGTC

AGAAGGAGAAACCTGTGGAATGCAG

CTGGGACTTTTTAGGAGTGTCTCTG

GAAACCTCAAAAGAGAAATCCTGCCAAAAGC

GGATTGCCATGGAAAGCATAACTGTGTATC

GAGTTATCTGGTGACAATATCGATCAAATTAAGG

AGTGAAAGGCTCCTGTGAAAAAGCTGC

CTAGCCCTGTGAGAGCTTCCTGTGC

CTAACAGCTGTCTGGGGTGGCATCCGTGGACTTCAC

GCTATGGGGACCCTATCCAAATCCTCACTGG

CCAATGGGAAAAGCCTTAGTTGAAC

GAACAGGTTTAAGGTCTAGGCTTTAGAGTTGGGGAAAGG

CAGGGGCTTTTCATATTTGTCAGAGGGCTTG

CCTTCTTCATAGTAAGCCGTACCCAGTGTTGGCAC

CTGACCATGGAAGCGAATGGGTTGGG

GCAGGCCGTTACTTACCAGGTAAGAGTC

CTGAGGCCTGCTGTGAACTGACTCTG

TTTCCTACAGCCACTGCGTCGCTTC

CTAGGCTATGTGTTCCAAGCCATCACC

GATGACATACATGGTTGAGGGTGGTG

GCATCTTGGCCCACAGCTCTTCAACAAGTTTGC

CCTTGAGGTTGAGGTGGGGGTGT

GGGAAGACGGTGACATTGCAG

CAGATGCTGGATGACTTTGGACCACATCGCTAC

GTGTCAAGAGAAGGGGCAGGGTCTATTTTAAAG

GTCTATCTATGCTCACCTTTGGCAG

CAAATGGCTTAGGCTTCACATAAATATCC

TTAACATCTCTCCTTTGATGTGTGTGTAAGCCTTAATG

CTATGTCAAGTGAGCTAGGTATAATGCATGAAGCC
$0.03(0.93-1.02)$

$0.06(0.91-1.10)$

$0.09(1.00-1.18)$

$0.05(0.90-1.06)$

$0.09(1.00-1.19)$

$0.03(0.94-1.00)$

$0.03(1.00-1.06)$

$0.05(1.00-1.10)$

$0.09(0.80-1.00)$

$0.07(0.86-1.00)$

$0.03(0.96-1.10)$

$0.08(1.00-1.16)$

$0.03(1.00-1.05)$

$0.07(0.84-1.00)$

$0.05(0.91-1.00)$

$0.07(1.00-1.14)$

$0.08(0.95-1.20)$

$0.04(0.97-1.05)$

$0.08(1.00-1.17)$

$0.06(0.86-1.00)$

$0.02(0.99-1.05)$

$0.02(0.96-1.05)$

$0.05(0.90-1.10)$

$0.06(0.91-1.10)$

$0.04(0.95-1.06)$

$0.04(0.92-1.05)$

$0.02(0.96-1.05)$

$0.03(0.96-1.09)$

$0.04(0.93-1.09)$

$0.04(0.92-1.14)$

$0.06(0.91-1.14)$

$0.07(0.86-1.10)$

$0.05(0.93-1.10)$

$0.05(0.94-1.09)$

$0.03(0.93-1.09)$

$0.05(0.92-1.09)$

$0.02(0.93-1.03)$

$0.02(0.94-1.03)$
GTTCTTGATAAGGATTCTTTATTAGGCTCAAGTCTC CACCTACTTTCAATGGGTATGAGTATTAGGTTGTC CTTTTAAACCAGCCTCCCTTTTTCTAAGCCTTTAGTG GATGTTTGATAATGCCAAAGATGATGGTGACTAACAGCTGTTTG GAGAGGAAGGAAGGTAAGGAGGC

GAAATCTTTCAGAGGAAGCTGCAAAACAAATG
$0.03(0.94-1.02)$ $0.05(0.87-1.02)$ $0.02(0.95-1.00)$ GTAGCGTATCTCAGCTAAAACCCTTATTACTTCATTGAATTAAC GAAAAGCGCCTTTGTGTCTAACTTGTAAAGACCTTTAATG CCAACTTTTCTGTGGACCGGTGG GTGTGTTTTCGAAAAGGCTGGGGTGAGCATTTCTGTTGTTCATG
$0.05(0.96-1.06)$

$0.04(0.94-1.06)$

$0.05(0.96-1.09)$ 
Table 1 Continued

\begin{tabular}{lllll}
\hline & Name & Upstream hybridizing sequence & Downstream hybridizing sequence & s.d. \\
\hline 7 & CPOX_3 & CATTTTGTGCTATGGGCGTGAGCTCTGTTATCCACC & CCAAGAATCCTCATGCTCCTACTATCCATTC & 0.03 (0.94-1.04) \\
8 & CPOX_4 & CAAGCAGTGGTGGTTGGTGGTGGATGTGAC & CTCACTCCAACATACTTGAATCAAGAAGACG & $0.04(0.94-1.05)$ \\
9 & CPOX_5 & GTACAGAGCTGTGCAGGGCTGTAGTTCCTTCTTACATTCC & CCTTGTGAAAAGCACTGTGATGACTCATTC & $0.05(0.93-1.07)$ \\
10 & CPOX_6 & CACAAAGTTGGCCTCTTCACTCCAGGATC & CAGAATTGAAGTATCTTGATGTCTTACCTCTAAC & $0.04(0.92-1.02)$ \\
11 & CPOX_7 & CATCCAAGGGACTGGGTGCGTTGATGCAG & GCAGAATGGCTGTGCAGGGGTTGGAG & $0.04(0.93-1.06)$ \\
12 & DOWN_1 & CCACTGTGTTCATGTCTCACTTTATGATTG & GGAGATTCTGAAGTTGGCAATGTCTACAATTCC & $0.02(0.96-1.01)$ \\
13 & DOWN_2 & CAATACCTTGTCTCACAGTTTAGGTGCCTC & CAGCCCCTAACCATTATTTGATTCAAG & $0.05(0.92-1.08)$ \\
14 & DOWN_3 & CCAAATCCTACCTGTTTAGACCAATCCAGG & GATATTATGTATGGCGACCTGGTGGTTAC & $0.05(0.94-1.06)$ \\
15 & DOWN_4 & CTACACTTCCACAGCTCCACCAG & GCATTACACTTTGAACCTAAATGGAGGC & $0.03(0.96-1.04)$ \\
16 & GPR15_1 & CTCTTCCTCCTCCTGGCAAAATCTTCT & GCATGAATGAAGGTGGAGAGAGC &
\end{tabular}

\section{PPOX}

1 KARCA_2

2 PFDN2 4

3 PFDN2_1

$4 \quad$ NIT 3

5 DEDD_4

6 UP_1

7 UFC1_1

8 USP213

9 PPOX_Prom

10 PPOX_2

11 PPOX 3

12 PPOX_4

13 PPOX_5

PPOX_6 PPOX_7

PPOX_8

PPOX_9

PPOX_10

PPOX_11

PPOX_12

PPOX_13

B4GAL 3

23 ADAM_9

24 NDUF_1
CCTATTGGTATGGCACCCTCCGCCCAAAACCTTTCAC TCTCATCCACACT

CCTTTGCCTGAAGCTGCTGTGTCAGTGTCTCAATGATCT TCTGTATCTAG

CCACAGGCTCCCCCTTCTCGCTAGTATTCCAGACCCAGG

CTGAGGCATTTGACTTCATTGCACGGGACCCTGCAGA

GACGCTACACC

CAGGTACGCAATGCTTTCCAGAATCCCTGCTCA

GCAGCTGCAATCC

CTCTGGGTCAACACATCATAGCCACATGTCCC

TACTAAATATTT

CGTGTTGTGTCTGAGATCCCGGTGC

CACCTGTTAATATCCAGCCCCGA

CTAACATCAAGGAGCTGTTTATGGAGCCGCCTCCCCGTG

CTAAGGTGAGTGCTCCACTTGTGC

CTAGTGGAGAGCAGTGAGCGTCTGGGAGGCT

CTGCATGCCCTACCCACTGGCCTCAGGTAA

CAAAGAGCCTGATGAGACTGTGC

CGCTCCTTAGTCCTAGTCTCACCCT

CTTTGCTTCCTCTGCAGGGCGGA

CTCTCAAATGTTTTCATGCTCTCAGGTATC

CTGAGTGCCATCACTGCAGTGTCTGTAGCTGTGG

CTCCAGGGATTTGGACATTTGGT

CAGCTGCTACACAATTAGGACTGAAGGAGATGCCGA

CСTCTCCTCTCTTCTCAGAACTGCATTC

CTGTTAATGACTGTATAGAGAGTGGGCGCCAGGCAGC

CTGAGGTTACTGTAGACATCACGAGGGTGAGAATAGTCAAAT

CCACATTGTTGTATCCGTACCTGTGTGGAAGGAGTA

GATGGGGAGCTCAG

CTGAGGGGACTGAGTGAGCTGCCTGAGAAAA

FECH

1 NARS_IVS2 CTGAAGACACACGGTTAGTAAAAGGCAGAACC

2 NARS_5 CAAATGAAGAGTGAATCCCGGGAAAGAAAGAGG TGAGTCCTGACC

3 NARS_10 CTTTTAAAAAATGCAGGCTCCAGGT

4 NARS_16

5 UP 1 CTTGGAACGATTCTTAACGTGGATTCTGAATAGGTATCACATCC CCAGTCCTGAGCCCCCTAGTGTAAT

FECH_PROM CCTCCAAGAAATGCACTTGCCAGGCTGG

FECH_IVS1 CGGTGCATATTTTATAAGTACTTTTGAAGTAATCCTCATCTCC

8 FECH_2 CCATGGAGGTGGAAGTCAGGTGCAGC

9 FECH_IVS2

10 FECH_3

11 FECH 4
CTTGACTAAAGCTTTCACTTCACATTCTGGAATAGAGG

CACCCTGGAAGAAAATAAGAAGTGTGACAAATCAAC

CTGGCACCATTCATCGCCAAACG
CTCGAAAAACAAAAGCCTAAGCAGATTTCTCA

GGCAATTTATCC

AGGACAAGTGACAGGGATTATATAAGGAATTCAGGACTCC

TGGGTACCCTGCTTCGCGTTAGCCACTCCTCACCTGC

TGTCTGAACCACTGGGTGGGAAACTTTTGGAAGAATAC

CCACCGTACTGAAAGGGCAGGGAAAGAACCGATGAGAC

TTTTACAAGTCAGTGGTAGTACCAGTTTAGAGTAAAG CAGATCTGTTC

TGAAGACTAACGCCGGACCCCGAGATCGTGAGTTGTGGG

GTGGGATCCAAGCTACCATTTGC

GACTGGCCTTAAGTGTCCCTATCTATTCTCCCTAC

CAGAGGGAGCTTCATTTAATGCTCTTCC

GGATTCGCTCCGTTCGAGGCCCTAATGGTGC

CACCAGCACCTCCGCTCCTTTTACTGTGCC

ACAGTTTTGCCCAGCGCCGCCTTGGACCTGAGG

TGACACTTGC

TAAGGTGGCGTCTCTAGCCATGGACAGTCTC

CCCCACAGCCAGACTCAGCACTCATTC

TCTAAGGGACAGCAGTCTGGAGGC

TGAATCTGCAGTACCAAGGAGCCCATCTGCCTGTCC

GCCATCTTCAGAAGATCCAGGAGTC

GCCACTGCTTGGTCCATCTACACAAGGTAAGTTGGGATAAAC

CCCAGTATACACTAGGTCACTGGCAAAAAC

AGTCAGTGTCCTGGGCACAGAACCTAACAGC

GTCGGTCCCTGATCTCGGCCAAATAGGGCACTGAGAC

TTCGGAAGC

GATCACCTGAATCCCCTCAGCCTTCTGAGC

GAGGGTAGGGAACAGGTAAGGAGGCATCAAGAG

GGAAGAAC

CAGGTTTGTATTCCGAAGCCCTCGTTCTTTCCAC

TTGCCATCGTGAACGTTGTTAAAGTTAGAACTCTGGCAAC

GGCCATGAGCTGAGTTGTGAC

GAGACGTGTGCTTATACCCTCGATTTGTCCAGCGTTGCAC CTGGATGGTTTTTGTTTAATACGGATGGCCAGC

CCTTCACTTTTCCTAATGCTCTTCAGTGAGTTTCAC

TATGTGATAGAAAAGTGCCATTATAGATTTCAA

TACTTGTGTATCTC

TGCAGCGGCCGTCACCACAGAAAC

GGTGTCAGACTGGGGCTTACTAATGCGCTATTCATCATGC CGTTGTATTTTATTTTATATAGGAAGCCGAAAACTG CCGAACCCCCAAGATTCAAGAGCAGTACC
$0.03(0.92-1.00)$

$0.03(0.94-1.03)$

$0.04(0.90-1.04)$

$0.03(0.90-1.00)$

$0.04(0.89-1.00)$

$0.03(0.93-1.00)$

$0.04(0.91-1.02)$

$0.03(0.93-1.04)$

$0.04(0.87-1.01)$

$0.04(0.93-1.08)$

$0.05(0.87-1.03)$

$0.02(0.94-1.00)$

$0.03(0.94-1.049$

$0.03(0.93-1.01)$

$0.03(0.95-1.04)$

$0.04(0.90-1.05)$

$0.04(0.89-1.03)$

$0.03(0.94-1.03)$

$0.03(0.93-1.04)$

$0.04(0.92-1.03)$

$0.05(0.90-1.05)$

$0.04(0.93-1.06)$

$0.04(0.88-1.01)$

$0.05(0.92-1.10)$

$0.02(0.98-1.04)$

$0.06(0.97-1.15)$

$0.03(0.97-1.07)$

$0.07(0.92-1.11)$

$0.03(0.99-1.07)$

$0.04(0.92-1.04)$

$0.04(0.92-1.02)$

$0.04(0.98-1.09)$

$0.03(0.97-1.05)$

$0.04(0.94-1.06)$

$0.03(0.99-1.06)$ 
Table 1 Continued

\begin{tabular}{|c|c|c|c|c|}
\hline & Name & Upstream hybridizing sequence & Downstream hybridizing sequence & s.d. \\
\hline 12 & FECH_IVS4 & CATTGAACAGCTGCTCTGAGGGTACGTAAGCTAGGCCAGTG & AGTGATGTGTTTTGGGCTCACTCTGGC & $0.03(0.97-1.05)$ \\
\hline 13 & FECH_5 & CTAGAAAGGGCTATTGCTTTCACACAG & TATCCACAGTACAGCTGCTCCACCACAGG & $0.03(0.97-1.06)$ \\
\hline 15 & FECH_7 & СTTTATCCTCCTTTCTTGTTACTCACTCAG & TGCTTTGCAGATCATATTCTAAAGGAACTG & $0.06(0.98-1.12)$ \\
\hline 16 & FECH_8 & CTCAGGAGGTAAGCGCCACTGTCCAAAAAGTCATG & GAAAGGCTGGAGTACTGCAACCCCTAC & $0.06(0.90-1.07)$ \\
\hline 17 & FECH_9 & CTCAAACAGACGAATCTATCAAAGGGCTTTGTGAGAGG & GGGAGGAAGAATATCCTCTTGGTTCCGATAGC & $0.02(0.95-1.01)$ \\
\hline 20 & DOWN_1 & $\begin{array}{l}\text { CTTTGGATAATGATAAAGGTCTCCTTGTGGCCGGG } \\
\text { TCGAATTTCAGG }\end{array}$ & GCCAGCCAAAACAAACATGCAAGGGAAATGCAG & $0.02(0.99-1.05)$ \\
\hline 21 & DOWN_2 & $\begin{array}{l}\text { CAAATGTTTAATCAGATCTGGGGTGGTATACTCTTGCC } \\
\text { TAACGCAATCTGAG }\end{array}$ & $\begin{array}{l}\text { GTTTTTCTGGAATTTTGGTCTGACTTTGGTTTG } \\
\text { GACAGTGCC }\end{array}$ & $0.03(0.96-1.05)$ \\
\hline 22 & DOWN_3 & САCTATTGTCTCCATCGCAACGGGAGATATAGAGACAGGTAA & $\begin{array}{l}\text { CTTTTCTCAAAGATGGGGCTGCTCCTCCTGAGT } \\
\text { CCAAGCTTGGCAAGGCC }\end{array}$ & $0.06(0.90-1.04)$ \\
\hline \multicolumn{5}{|c|}{ CTRL } \\
\hline 1 & DEFB129_2F & GCCACCATCAGCACTATGACC & CCAGGACAGATCACATACACTGC & $0.01(0.99-1.02)$ \\
\hline 2 & DACH_9F & $\begin{array}{l}\text { CATTTAAGACCCTGAGACTATCTGTTGAAGCTG } \\
\text { CCTGTTTTAGCGT }\end{array}$ & CTGTTCTGCTTGTTCACGCCGTTTCGTCTCAAACTC & $0.05(0.93-1.05)$ \\
\hline 3 & ILKAP_7F & $\begin{array}{l}\text { CTGGCTGGAAGCTTGTTTAAGGAACTCTTCATCAG } \\
\text { TATGCTTGAAAGTGT }\end{array}$ & $\begin{array}{l}\text { CCAAAAGGCATCTCTTCACGGTTTTCTCTACAC } \\
\text { TGATTACATCTCC }\end{array}$ & $0.05(0.90-1.07)$ \\
\hline
\end{tabular}

Abbreviation: s.d., standard deviation.

the genomic region was amplified. Each probe was designed at least corresponding to each coding exon of heme gene, and if a high GC percentage in exon 1 did not allow for positioning the probe, it was located in the upstream promoter region. In the presence of heme genes longer than $15 \mathrm{~kb}$, the probes were also located in the longest introns. With regard to the flanking genes, the probes were designed corresponding to coding exons or intergenic regions, and at any rate spaced at $10 \mathrm{~kb}$.

Each probe was designed as two different oligonucleotides, each containing an unique sequence annealing to the target DNA and a common tag corresponding to MAPH primers, which have no complementarities in the whole human genome (forward tag 5'-GGCCGCGGGAATTCGATT-3' reverse tag $5^{\prime}$-CACTAGTGAATTCGCGGC- ${ }^{\prime}$ ). Unique sequences were identified using the BLAT program (http://genome.ucsc.edu), and care was taken to ensure that no known sequence variants were present in the probe-annealing sites. Using the RAW program (MRC-Holland, Amsterdam, the Netherlands), the hybridizing regions of each oligonucleotide were chosen such that the melting temperature was at least $67.5^{\circ} \mathrm{C}$ with a GC percentage between 30 and $63 \%$. The entire oligonucleotides (unique sequence plus tag) were checked in DNA folding form (http://frontend.bioinfo.rpi.edu/applications/mfold/cgi-bin/dnaform1.cgi) to have $\Delta \mathrm{G}$ (secondary structure) $\geqslant 0$ with parameters set on $\left[\mathrm{Na}^{+}\right]=0.35 \mathrm{M}$ and $\mathrm{T}=60^{\circ} \mathrm{C}$.

The ligation site was chosen to be flanked by no more than three guanine and/or cytosine bases to ensure efficient ligation, and $\mathrm{A}$ and $\mathrm{T}$ bases were never permitted to be adjacent to the universal primer tags to ensure efficient amplification. The probes within each set were designed to produce PCR products differing by $2 \mathrm{bp}$ in length to allow separation in the size range of 80-132 bp using capillary electrophoresis on an ABI PRISM 310 sequencer (Applied Biosystems, Foster City, CA, USA). The probes corresponding to the three internal control genes (ILKAP, DACH and DEFB129) had a fixed length of 132 nucleotides (nt), $118 \mathrm{nt}$ and $80 \mathrm{nt}$, respectively. Each probe was chemically synthesized by Eurofins MWG-Operon (Ebersberg, Germany) as two different unlabelled oligonucleotides. The downstream oligo of each probe was $5^{\prime}$-phosphorylated to allow ligation and purified by reversed-phase high performance liquid chromatography, whereas the upstream oligo was purified by HPSF (high purity salt free) method. Probe mixes were prepared by combining each oligonucleotide so that all were present at a final concentration of $4 \mathrm{fmol} \mathrm{I}^{-1}$. The seven probe mixes are available on request.

\section{MLPA reaction}

Reagents for MLPA were purchased from MRC-Holland. The MLPA reactions were performed, as previously described. ${ }^{16,17}$ In brief, approximately $100 \mathrm{ng}$ of genomic DNA (Puregene kit, Qiagen, Hilden, Germany) in a final volume of $5 \mu \mathrm{l}$ was heated for $5 \mathrm{~min}$ at $98^{\circ} \mathrm{C}$. After cooling to room temperature, $1.5 \mu \mathrm{l}$ of the probe mix and $1.5 \mu \mathrm{l}$ of SALSA hybridization buffer (MRC-Holland) were added to each sample, followed by heat denaturation $\left(1 \mathrm{~min}\right.$ at $\left.95^{\circ} \mathrm{C}\right)$ and hybridization $\left(16 \mathrm{~h}\right.$ at $\left.60^{\circ} \mathrm{C}\right)$. Ligation was performed by adding $32 \mu \mathrm{l}$ of ligation mix at $54^{\circ} \mathrm{C}$ for $15 \mathrm{~min}$ and the reaction was stopped by incubating for $5 \mathrm{~min}$ at $98^{\circ} \mathrm{C}$. PCR amplification was carried out for 35 cycles in a final volume of $25 \mu \mathrm{l}$, adding dNTPs mix to a final concentration of $200 \mu \mathrm{M}$, and both the MAPH-F and MAPH-R primers to a final concentration of $200 \mathrm{nM}$, with MAPH-F being labelled with 6-FAM. A total of $0.75 \mu \mathrm{l}$ of water, $13.5 \mu \mathrm{l}$ of HiDi formamide (Applied Biosystems) and $0.5 \mu \mathrm{l}$ of internal size standard (500 ROX; Applied Biosystems) were added to $0.75 \mu$ l of PCR reaction. The mixes were denatured for $2 \mathrm{~min}$ and cooled on ice before being separated by capillary electrophoresis on the ABI Prism 310 sequencer, with the following settings: filter set D; capillary length $47 \mathrm{~cm}$; POP-6 polymer; injection time $10-30 \mathrm{~s}$; injection voltage $3.0 \mathrm{kV}$; run temperature $60^{\circ} \mathrm{C}$; run time $25-30 \mathrm{~min}$

\section{Data analysis}

For quantitative analysis of probes, which were successfully amplified, the trace data produced by the capillary electrophoresis were retrieved using the accompanying software (GeneMapper, Applied Biosystems) and subsequently exported to Excel (Microsoft) for further analysis. The data of each probe set were analyzed separately. Three probes for unlinked loci were included in each probe set as a reference in each sample (internal control). The height of each gene-specific probe peak was divided by the sum of the heights of the three 

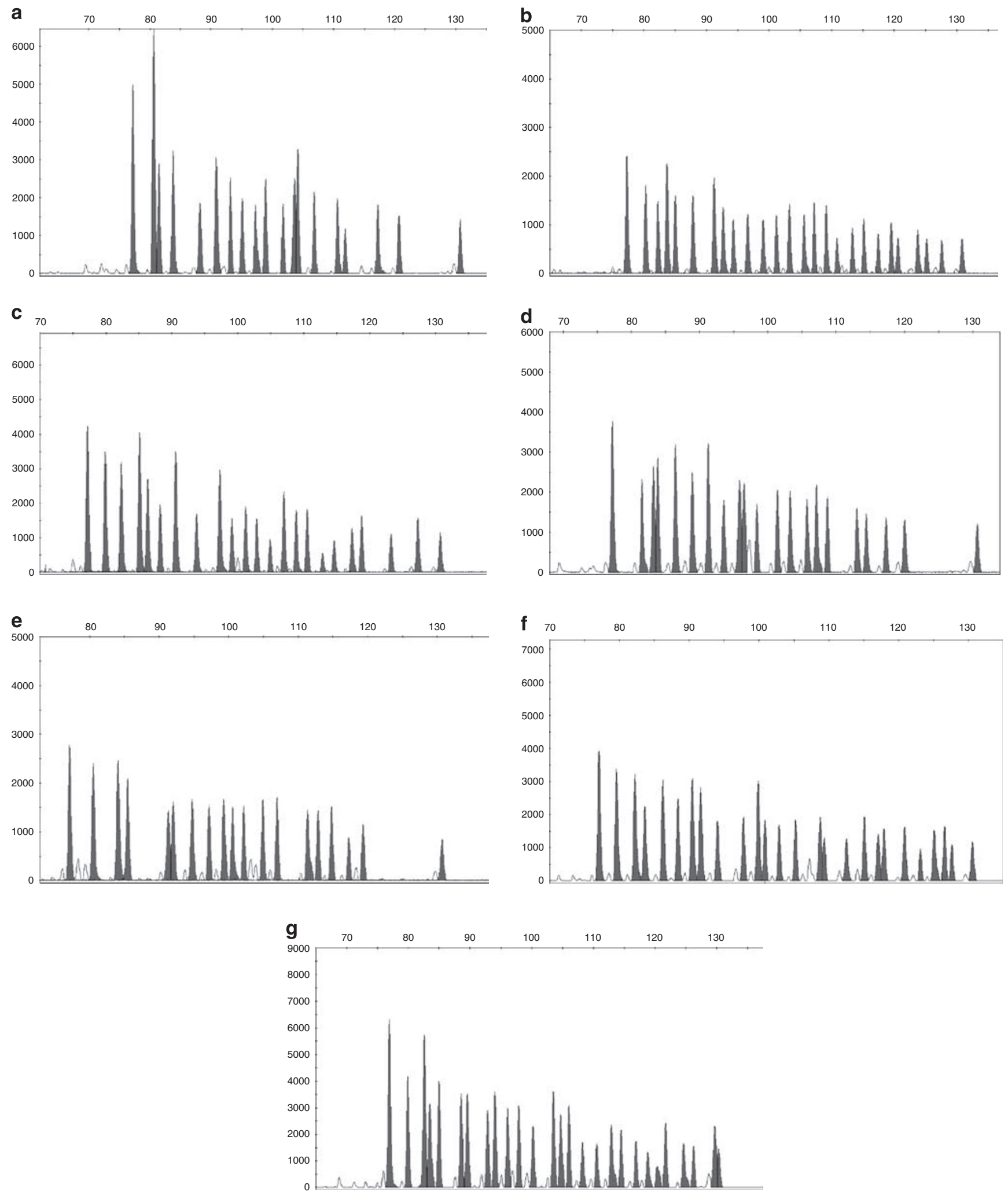

Figure 1 Peak patterns. (a) 19 probes in ALAD set; (b) 26 probes in HMBS set; (c) 23 probes in UROS set; (d) 21 probes in UROD set; (e) 27 probes in PPOX set; (f) 19 probes in CPOX set; and (g) 27 probes in FECH.

reference probe peaks to obtain a ratio. The median ratio for each probe across all wild-type samples (external control) was calculated and was used as the divisor to normalize each probe value to 1.0 dosage quotient (DQ), corresponding to a copy number of two. The resulting data were then represented by a scatter plot, and the thresholds for deletions and duplications were set at 0.8 and 1.2 , respectively. 
CTRL

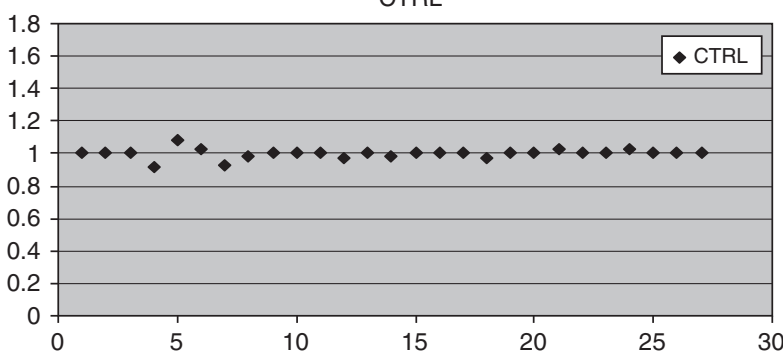

CTRL

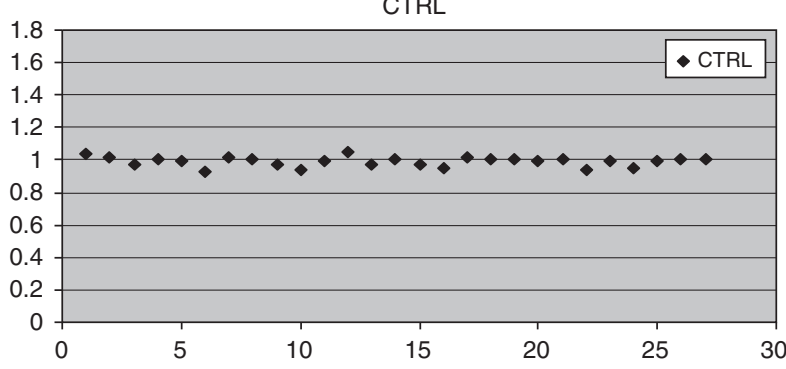

844

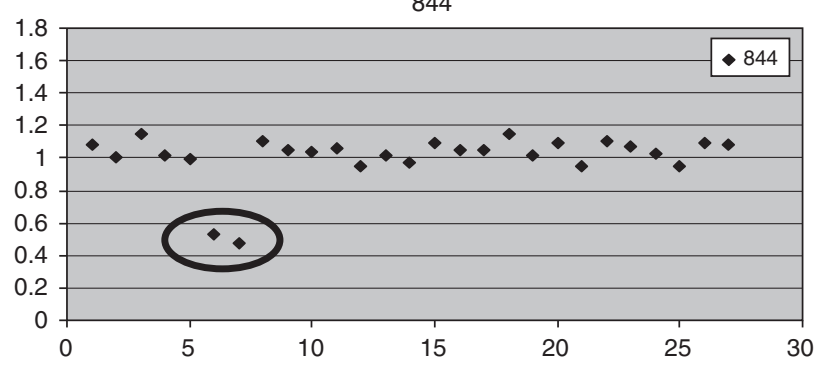

CTRL

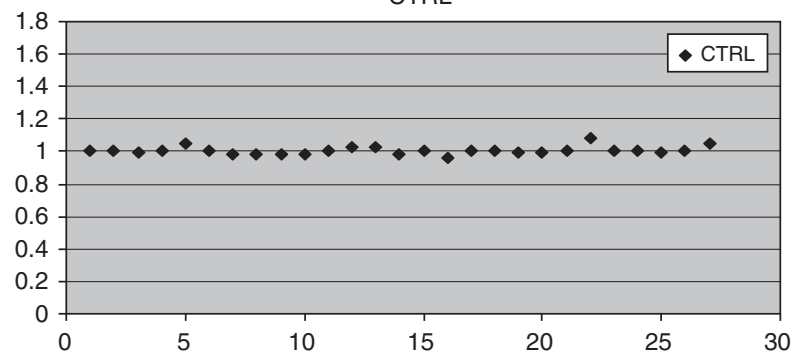

909

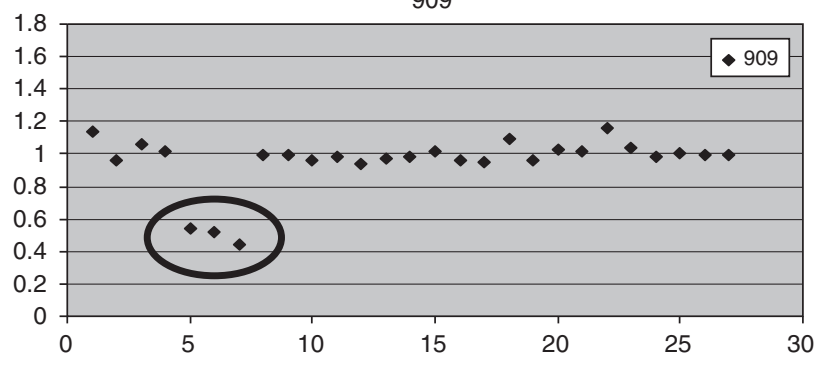

399

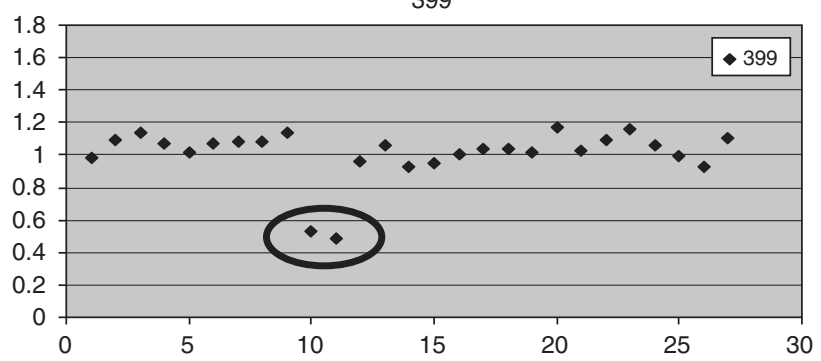

Figure 2 Scatter plots of FECH experiment. The first three plots marked as CTRL correspond to normal participants, plots 909 and 844 represent two known $\mathrm{FECH}$ promoter deletions of different lengths, the plot marked as 399 shows the deletion of exons 3 and 4 .

\section{RESULTS}

The probes were tested on seven control samples to assess their reliability and consistency. Each set was tested at least thrice. All synthesized probes generated an amplification product with fluorescent peaks within the required intensity range (Figure 1) and, comparing independent samples, all showed standard deviation of lower than $10 \%$ (Table 1). Greater standard deviation values (up to $20 \%$ ) were observed when comparisons were performed between samples from different sources (that is, methods of DNA extraction). Comparing both identical input samples and independent duplicate samples, the reproducibility of the methods was very satisfactory with standard deviation $<5 \%$. Using three internal controls having lengths of $80 \mathrm{nt}, 118 \mathrm{nt}$ and $132 \mathrm{nt}$ for normalization, we obtained a very small DQ variation of probe across the genes (DQ ranged between 0.9 and 1.1). The sizes of the control probes - small, medium and largereduce the bias due to amplification of larger products. Greater DQ variations (from 0.8 up to 1.2 ) resulted in cases of very poor DNA quality. To prove the validity of the method, DNA from six patients carrying deletions, which had already been characterized by other techniques, was used as positive controls for HMBS and FECH. In all cases, the deletions were clearly confirmed (Figures 2 and 3). DNA of patients carrying point mutations was used to test the specificity of binding, except for the ALAD set. Under our experimental conditions, a mismatch at the $3^{\prime}$-end of the oligonucleotides, and also at 2-3 nt before, completely prevented the appearance of probe amplification products. Mismatches at the middle of the oligonucleotides had no effects (Figure 3).

\section{DISCUSSION}

In this paper, we describe a novel approach for molecular diagnosis of porphyrias. The MLPA is an attractive method compared with more complicated and time-consuming techniques such as Southern blot and gene dosage. It is based on comparative quantification of specifically bound probes that are amplified by PCR using universal primers. These alternative techniques have advantages, in that multiplexing numerous targets becomes much easier. Moreover, the use of one fluorescent primer reduces the cost compared with using fluorescent probes for each target. In the original description, the probes were generated by cloning into specially developed M13 vectors, but a simpler method, which relies on chemical synthesis of oligonucleotides, has also been described. ${ }^{18,19}$ In this case, the synthesis length, limited to a maximum of 70-75 nt, reduces the number of probes that could be used in a single probe set, even though the use of multiple fluorophores avoids this problem. ${ }^{17}$ We chose the chemical synthesis of probes because it allows for cheaper and more rapid probe production, and their use in these assays was robust and reliable. 
CTRL

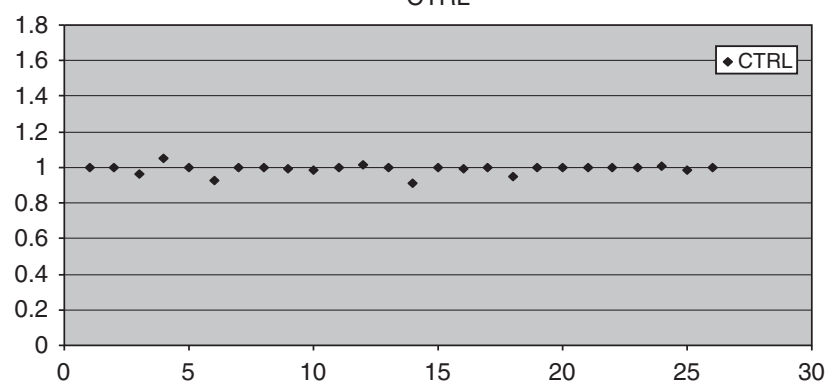

CTRL

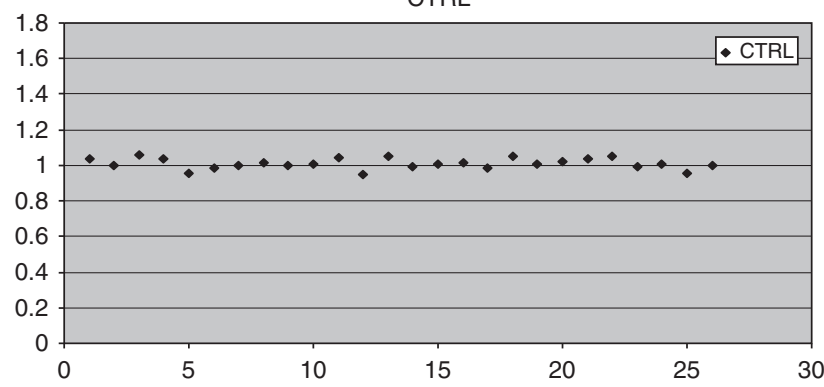

526

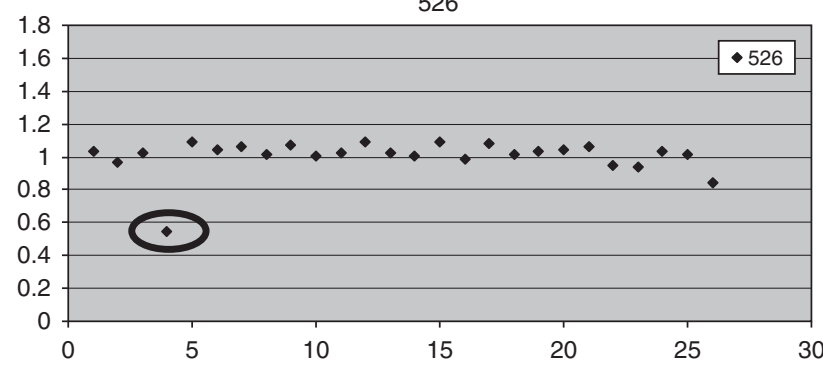

CTRL

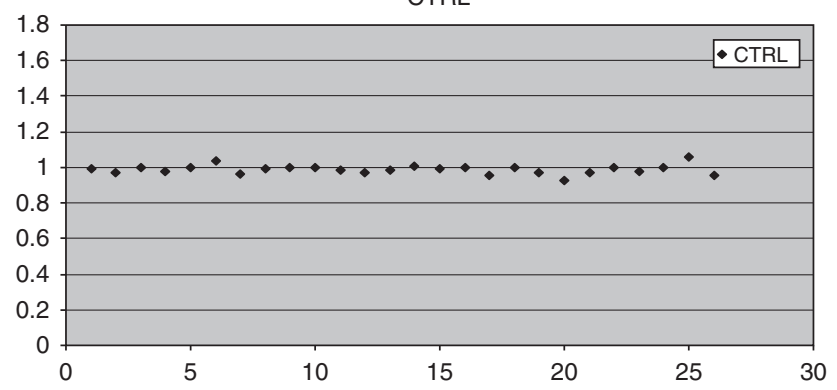

496

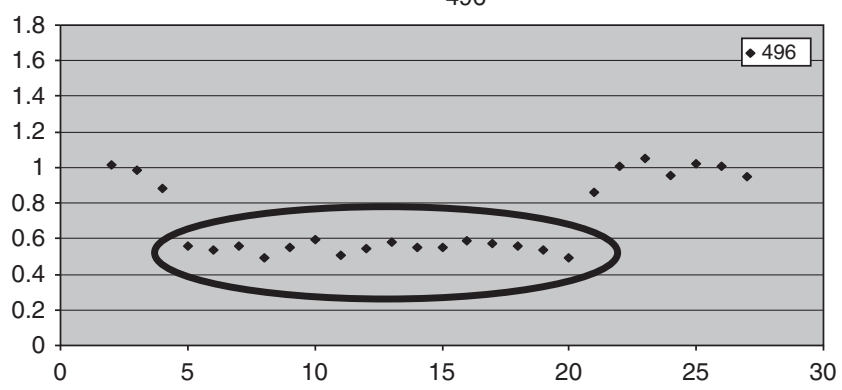

642

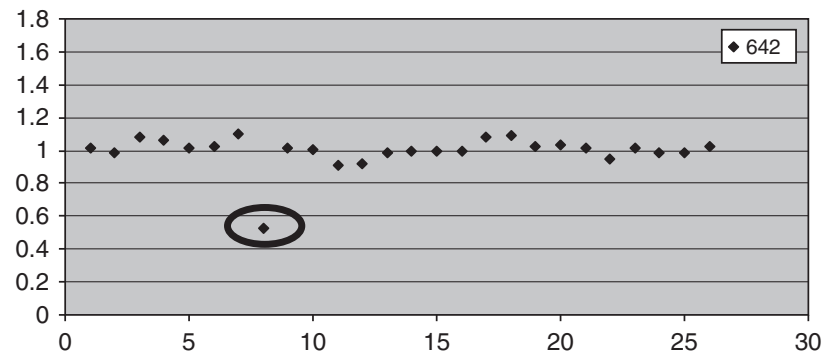

Figure 3 Scatter plots of HMBS experiment. The first three plots marked as CTRL correspond to normal participants, plot 496 represents a deletion of entire HMBS gene and two flanking downstream genes, the plots marked as 526 and 642 show the absence of probe amplification products due to mismatch at 3 '-end of the oligonucleotides.

The majority of probes validated on seven wild-type controls showed standard deviation between 0.03 and 0.06 compared with those of commercially available cloned probes. ${ }^{16}$ The size range of $80-132 \mathrm{bp}$ was used for the different probes of each set, with up to 27 probes being combined within a single probe mix, confirming that individual oligonucleotides of up to $70 \mathrm{bp}$ in length can be used effectively. The main limitation of this technique is the need for good quality and accurately quantified DNA, a factor that affects cloned and synthetic probes alike. Point substitutions in the genome could interfere with probe annealing and ligation, causing a loss of probe signal and a false positive MLPA result. Even though we accurately excluded annealing sites containing known sequence variants (SNPs) in the probe design, the presence of point mutation in DNA of patients cannot be prevented. For this reason, it is mandatory to perform standard mutational analysis before MLPA. In conclusion, the MLPA assay was shown to be an efficient method for ascertaining copy number changes at multiple loci of heme genes, as all the known tested deletions were confirmed. In the recent years, deletions of single genes or parts of them have been reported as responsible for different forms of Porphyria. ${ }^{11,13,14}$ Chromosome deletions of megabases have also been reported in late-onset porphyria caused by clonal expansion in the myelodysplastic process, ${ }^{20}$ suggesting that heme genes are prone to this type of molecular abnormality. Considering the high molecular heterogeneity and the technical difficulties in determining insertions, deletions or complex rearrangements of a few kilobase pairs, it is quite possible that patients with clinical symptoms of porphyria remain undiagnosed even in specialized centers, because of undetected mutations. Undiagnosed patients with porphyrias are often exposed to useless treatments, including surgery, that may be fatal (many drugs, commonly used in clinical practice or anesthesia, have porphyrinogenic effects). Late diagnosis may be responsible for increased morbidity, high health-care costs and poor quality of life. Considering that acute porphyric attacks can be prevented by avoiding triggering factors, to prevent clinical manifestations, it is mandatory to make available all the procedures that can increase the possibility of a correct and early diagnosis. MLPA properly designed for porphyria genes was shown to be a reliable and reproducible method for detecting deletions that so far have been difficult to identify.

\section{ACKNOWLEDGEMENTS}

This work was supported by FIRST 2006/07 and PRIN 2006 ProjectsUniversity of Milan, and Maggiore Policlinico, Mangiagalli and Regina Elena IRCCS Foundation grants. We are very grateful to the blood donors, the patients and referring physicians for their cooperation. 
1 Kappas, A., Sassa, S., Gelbraith, R. \& Nordmann, Y. In The Metabolic Basis Of Inherited Disease (eds. Scriver, C., Beaudet, A., Sly, WS. \& Valle, D.) pp. 21032159 (McGraw-Hill, New York, 1995).

2 Kauppinen, R. Porphyrias. Lancet 365, 241-252 (2005).

3 Thunell, S., Harper, P., Brock, A. \& Petersen, N. E. Porphyrins, porphyrin metabolism and porphyrias. II. Diagnosis and monitoring in the acute porphyrias. Scand. J. Clin. Lab. Invest. 60, 541-559 (2000).

4 Thunell, S., Harper, P. \& Brun, A. Porphyrins, porphyrin metabolism and porphyrias. IV. Pathophysiology of erythyropoietic protoporphyria-diagnosis, care and monitoring of the patient. Scand. J. Clin. Lab. Invest. 60, 581-604 (2000).

5 McDonagh, A. F. \& Bissell, D. M. Porphyria and porphyrinology - the past fifteen years. Semin. Liver Dis. 18, 3-15 (1998).

6 Sassa, S., Granick, S., Bickers, D. R., Bradlow, H. L. \& Kappas, A. A microassay for uroporphyrinogen I synthase, one of three abnormal enzyme activities in acute intermittent porphyria, and its application to the study of the genetics of this disease. Proc. Natl. Acad. Sci. USA 71, 732-736 (1974).

7 Meissner, P. N., Dailey, T. A., Hift, R. J., Ziman, M., Corrigall, A. V., Roberts, A. G. et al. A R59W mutation in human protoporphyrinogen oxidase results in decreased enzyme activity and is prevalent in South Africans with variegate porphyria. Nat. Genet. 13, 95-97 (1996).

8 Hrdinka, M., Puy, H. \& Martasek, P. May 2006 update in porphobilinogen deaminase gene polymorphisms and mutations causing acute intermittent porphyria: comparison with the situation in Slavic population. Physiol. Res. 55 (Suppl 2), S119-S136 (2006).

9 Aurizi, C., Schneider-Yin, X., Sorge, F., Macri, A., Minder, E. I. \& Biolcati, G. Heterogeneity of mutations in the ferrochelatase gene in Italian patients with erythropoietic protoporphyria. Mol. Genet. Metab. 90, 402-407 (2007).

10 Martinez di, M. F., Di, P. E., Patti, E., Tavazzi, D., Danielli, M. G., Biolcati, G. et al. Molecular characterization of porphyrias in Italy: a diagnostic flow-chart. Cell Mol. Biol. (Noisy-le-grand) 48, 867-876 (2002).
11 Di Pierro, E., Besana, V., Moriondo, V., Brancaleoni, V., Tavazzi, D., Casalgrandi, G. et al. A large deletion on chromosome 11 in acute intermittent porphyria. Blood Cells Mol. Dis. 37, 50-54 (2006).

12 Di Pierro, E., Brancaleoni, V., Moriondo, V., Besana, V. \& Cappellini, M. D. Co-existence of two functional mutations on the same allele of the human ferrochelatase gene in erythropoietic protoporphyria. Clin. Genet. 71, 84-88 (2007).

13 Wood, L. H., Whatley, S. D., McKenna, K. \& Badminton, M. N. Exonic deletions as a cause of erythropoietic protoporphyria. Ann. Clin. Biochem. 43, 229-232 (2006).

14 Whatley, S. D., Mason, N. G., Holme, S. A., Anstey, A. V., Elder, G. H. \& Badminton, M. N. Gene dosage analysis identifies large deletions of the $\mathrm{FECH}$ gene in $10 \%$ of families with erythropoietic protoporphyria. J. Invest. Dermatol. 127, 2790-2794 (2007).

15 Di Pierro, E., Brancaleoni, V., Besana, V., Ausenda, S., Drury, S. \& Cappellini, M. D. A 10376 bp deletion of $\mathrm{FECH}$ gene responsible for erythropoietic protoporphyria. Blood Cells Mol. Dis. 40, 233-236 (2008).

16 Schouten, J. P., McElgunn, C. J., Waaijer, R., Zwijnenburg, D., Diepvens, F. \& Pals, G. Relative quantification of 40 nucleic acid sequences by multiplex ligation-dependent probe amplification. Nucleic Acids Res. 30, e57 (2002).

17 White, S. J., Vink, G. R., Kriek, M., Wuyts, W., Schouten, J., Bakker, B. et al. Two-color multiplex ligation-dependent probe amplification: detecting genomic rearrangements in hereditary multiple exostoses. Hum. Mutat. 24, 86-92 (2004).

18 Stern, R. F., Roberts, R. G., Mann, K., Yau, S. C., Berg, J. \& Ogilvie, C. M. Multiplex ligation-dependent probe amplification using a completely synthetic probe set. Biotechniques 37, 399-405 (2004).

19 Harteveld, C. L., Voskamp, A., Phylipsen, M., Akkermans, N., den Dunnen, J. T., White, S. J. et al. Nine unknown rearrangements in $16 \mathrm{p} 13.3$ and $11 \mathrm{p} 15.4$ causing alpha- and beta-thalassaemia characterised by high resolution multiplex ligation-dependent probe amplification. J. Med. Genet. 42, 922-931 (2005).

20 Aplin, C., Whatley, S. D., Thompson, P., Hoy, T., Fisher, P., Singer, C. et al. Late-onset erythropoietic porphyria caused by a chromosome $18 \mathrm{q}$ deletion in erythroid cells. J. Invest. Dermatol. 117, 1647-1649 (2001). 